\title{
The Beta Power Exponential Distribution
}

\author{
Kazeem A. Adepoju, Chukwu, A.U \\ Department of Statistics, University of Ibadan, Nigeria \\ Min Wang \\ Michigan Technology University, USA
}

\begin{abstract}
A new three-parameter beta power distribution is introduced and studied. We derive formal expressions for its moments, generating function and Cumulative density function. The maximum likelihood estimation of the model parameters was also conducted. In the end, the superiority of the new distribution over the exponentiated exponential was made by means of data set.

Key words: Exponential distribution, Beta power exponential, exponentiated exponential, maximum likelihood
\end{abstract}

\section{Introduction}

In recent time probability distributions play a significant role in modeling naturally occurring phenomena. By this, different kinds of distribution functions have been propounded and defined extensively in the statistical literature in modeling many real life phenomena. Various distributions emerge from the need to capture some naturally occurring events. For instance, Pareto distribution has been justified in modeling the size of companies, family income size, and population size among others. Poisson distribution can be used to measure the occurrence of rare events; Weibull and gamma have been identified as good distributions in modeling the amount of rainfall in African among other functions.

Probability distributions revealed lately involve compounded beta family of distribution which include beta-normal Eugene (2002); beta-Gumbel [Nadarajah and Kotz (2004)], beta-Weibull [Famoye (2005)], beta-exponential [Nadarajah and Kotz (2006)]; beta- Rayleigh [Akinsete and Lowe (2008)]; beta-Laplace [Kozubowski and Nadarajah (2008)]; beta-Pareto [Akinsete (2008)]; Kumaraswamy Pareto [Marcelo Bourguignon, etal (2012)], beta Nakagami [Shittu and Adepoju(2013)] among others.

\section{The New Distribution}

A random variable $\mathrm{X}$ is distributed as the Beta power exponential distribution if it satisfies the probability density function given as follows;

$$
f(x)=\frac{\lambda}{\beta(a, b+1)}\left(1-\ell^{-\lambda x}\right)^{a-1}\left(\ell^{-\lambda x}\right)^{b+1}
$$

This can be showed to be a proper density function as below

Corresponding author: Kazeem A. Adepoju, Department of Statistics, University of Ibadan, Nigeria, E-mail: ka.adepoju@.ui.edu.ng.

Chukwu, A.U, Department of Statistics, University of Ibadan, Nigeria, E-mail: au.chukwu@ui.edu.ng.

Min Wang, Michigan Technology University, USA, E-mail: minwang@mtu.edu. 
If we let $y=\ell^{-\lambda x} \quad \frac{d y}{d x}=-\lambda \ell^{-\lambda x}$; we have

$$
\begin{aligned}
& \frac{-\lambda}{\beta(a, b+1)} \int(1-y)^{a-1} \frac{y^{b} d y}{\lambda} \\
& \frac{1}{\beta(a, b+1)} \int_{0}^{1} y^{b}(1-y)^{a-1} d y
\end{aligned}
$$

Let $M=1-y \Rightarrow Y=1-M$

$$
\begin{gathered}
\frac{d m}{d y}=-1 \Rightarrow d y=-d m \\
\frac{1}{\beta(a, b+1)} \int_{0}^{1} m^{a-1}(1-m)^{b} d m \\
\frac{\beta(a, b+1)}{\beta(a, b+1)}=1
\end{gathered}
$$

This verifies that distribution in Eq. (1) is indeed a proper density function.

\section{Cumulative Function}

Here we seek to the obtain the cumulative function of the new distribution, this is as follows from Eq. (1), we have

$$
\begin{aligned}
f(x) & =\frac{1}{\beta(a, b+1)} \int_{0}^{x} \lambda \ell^{-\lambda y}\left(1-\ell^{-\lambda y}\right)^{a-1} \ell^{-\lambda y b} d y \\
& =\frac{1}{\beta(a, b+1)} \int_{\alpha}^{x}\left(\ell^{-\lambda y}\right)^{b-1}\left(1-\ell^{-\lambda y}\right)^{a-1} d y
\end{aligned}
$$

Let $U=\lambda y \quad \frac{d u}{d y}=\lambda \Rightarrow d y=\frac{d u}{\lambda}$

$$
F(x)=\frac{1}{\beta(a, b+1)} \int_{0}^{\lambda x}\left(\ell^{-U}\right)^{b-1}\left(1-\ell^{-U}\right)^{a-1} d u .
$$

If $a>0$ is real non-integer we have

$$
(1-K)^{a-1}=\sum_{j=0}^{\infty}(-1)^{j} \frac{\sqrt{\Gamma(a)} K^{j}}{\sqrt{\Gamma(a-j)} J_{i}^{i}}
$$

Then using Eq. (3) in Eq. (2), we have 


$$
=\left.\frac{1}{\beta(a, b+1)} \sum_{j=0}^{\infty} \frac{\left.(-1)^{j}\right)(a)}{\sqrt{(a-j)} j !} \frac{\ell^{-u(b-1+j)}}{-(b+j-1)}\right|_{0} ^{\lambda x}
$$

Finally, using the fact that

$$
\begin{gathered}
\beta(a, b)=\frac{\sqrt{(a)} \sqrt{(b)}}{\sqrt{(a+b)}}, \text { we have } \\
F(x)=\frac{\sqrt{(a+b+1)}}{\sqrt{(b+1)}} \sum_{j=0}^{\infty}(-1)^{j} \frac{\left[1-\ell^{-\lambda x(b+j-1)}\right]}{\sqrt{(a-j)} j !(b+j-1)}
\end{gathered}
$$

For positive real non-integer $a$, Eq. (5), can be used for further analytical and / or numerical studies.

For integer $a$, Eq. (4) can be written as

$$
F(x)=\frac{1}{\beta(a, b+1)} \sum_{j=0}^{a-1}\left(\begin{array}{c}
a-1 \\
j
\end{array}\right)(-1)^{j} \frac{\left[1-\ell^{-\lambda x(b+j-1)}\right]}{(b+j-1)}
$$

From the wolfram Function site for integer $a$

$$
I_{y}(a, b)=1-\frac{(1-y)^{b}}{\sqrt{(b)}} \sum_{j=0}^{a-1} \frac{\sqrt{(b+j} y^{j}}{j !}
$$

and for integer $b$

$$
I_{y}(a, b)=\frac{y^{a}}{\sqrt{(a)}} \sum_{j=0}^{b-1} \frac{\sqrt{(a+j}(1-y)^{j}}{j !}
$$

Then, if $a$ is integer Eq. (5) can also be written as

$$
F(x)=1-\ell \frac{\left(\ell^{-\lambda x}\right)^{b}}{\sqrt{(b)}} \sum_{j=0}^{a-1} \frac{\overline{(b+j)}\left(1-\ell^{-\lambda x}\right)^{j}}{j !}
$$

For integer value of $b$, we have

$$
F(x)=\frac{\left(1-\ell^{-\lambda x}\right)^{a}}{\sqrt{(a)}} \sum_{j=0}^{b-1} \frac{\overline{(a+j})\left(\ell^{-\lambda x j}\right)}{j !}
$$

\section{Moments and Generating Function}

In this section, we derive the $r$ th moment about the origin and the moment generating function for a random variable $\mathrm{X}$ having the density function in Eq. (1). It is convenient to introduce

$$
S_{d, b, a}=\int_{0}^{\infty} x^{d-1} \ell^{-x(b+1)}\left(1-\ell^{-x}\right)^{a-1} d x
$$


Let $w=e^{-x} \quad \mid n w=-x$

$$
\begin{gathered}
x=|\operatorname{Inw}| \quad \frac{d x}{d w}=\frac{1}{w} \\
d x=\frac{d w}{w} \\
S_{d, b, a}=\int_{0}^{\infty}|\operatorname{In} w|^{d-1} w^{b+1}(1-w)^{a-1} \frac{d w}{w} \\
S_{d, b, a}=\int_{0}^{\infty}|\operatorname{In} w|^{d-1} w^{b}(1-w)^{a-1} d w
\end{gathered}
$$

If $a>0$ and is non-integer we have

$$
=\sqrt{(a)} \sum_{j=0}^{\infty} \frac{(-1)^{j}}{\sqrt{(a-j)} j_{i}^{i}} \int_{0}^{1}|\operatorname{In} w|^{d-1} w^{b+j} d w
$$

Also, for real $p>-1$, and real $q$, we have

$$
\int_{0}^{1} x^{p}|\operatorname{In} x|^{q} d x=\frac{\overline{(1+q)}}{(1+p)^{q+1}}
$$

Then,

$$
\begin{gathered}
\int_{0}^{1} w^{b+j}|\operatorname{Inw}|^{d-1} d w=\frac{\sqrt{1+d-1}}{(1+b+j)^{d-1+1}} \\
=\frac{\sqrt{d}}{(b+j-1)^{d}} \\
\int_{0}^{\infty} x^{d-1} f(x) d x=\sqrt{(a)} \sum_{j=0}^{\infty} \frac{(-1)^{j} \quad \sqrt{d}}{\sqrt{(a-j)} j !(b+j+1)^{d}}
\end{gathered}
$$

Finally, we have

$$
\begin{gathered}
\int_{0}^{\infty} x^{d-1} e^{-x(b+1)}\left(1-e^{-x}\right)^{a-1} d x= \\
\sqrt{(a)} \sqrt{(d)} \sum_{j=0}^{\infty} \frac{(-1)^{j}}{\sqrt{(a-j)} j !(b+j+1)^{d}}
\end{gathered}
$$

If we let $a>0$ be an integer then from ( )

$$
\int_{0}^{\infty} x^{d-1} f(x) d x=\sum_{j=0}^{a-1}\left(\begin{array}{c}
a-1 \\
j
\end{array}\right)(-1)^{j} \int_{0}^{1}|\operatorname{In} w|^{d-1} \quad w^{b+j} d w
$$


By using ( ), we have

$$
\int_{0}^{\infty} x^{d-1} f(x) d x=\sum_{j=0}^{a-1} \frac{\left.\left(\begin{array}{c}
a-1 \\
j
\end{array}\right)(-1)^{j}\right)(d)}{(b+j+1)^{d}}
$$

which simplifies to

$$
\begin{gathered}
\int_{0}^{\infty} x^{d-1} e^{-x(b+1)}\left(1-e^{-x}\right)^{a-1} d x \\
=\sqrt{(d)} \sum_{j=0}^{a-1} \frac{\left(\begin{array}{c}
a-1 \\
j
\end{array}\right)(-1)^{j}}{(b+j+1)^{d}}
\end{gathered}
$$

Then mgf of X can be written

$$
\begin{aligned}
& M_{(t)}=\frac{\lambda}{\beta(a, b+1)} \int_{0}^{\infty} e^{t x}\left(e^{-\lambda x}\right)^{b+1}\left(1-e^{-\lambda x}\right)^{a-1} d x \\
& =\frac{\lambda}{\beta(a, b+1)} \sum_{r=0}^{\infty} \frac{t^{r}}{r !} \int_{0}^{\infty} x^{r}\left(e^{-\lambda x}\right)^{b+1}\left(1-e^{-\lambda x}\right)^{a-1} d x
\end{aligned}
$$

Considering $\int_{0}^{\infty} x^{r}\left(e^{-\lambda x}\right)^{b+1}\left(1-e^{-\lambda x}\right)^{a y} d x$

Let $f=e^{-\lambda x}$

$$
\begin{gathered}
\operatorname{Inf}=-\lambda x \\
x=\frac{1}{\lambda}|\operatorname{Inf}| \\
\frac{d x}{d f}=\frac{1}{\lambda f} \Rightarrow d x=\frac{d f}{\lambda f} \\
\int_{0}^{\infty}\left[\frac{1}{\lambda}|\operatorname{Inf}|\right]^{r} f^{b+1}(1-f)^{a-1} \frac{d f}{\lambda f} \\
\frac{1}{\lambda^{r+1}} \int_{0}^{\infty}|\operatorname{Inf}|^{r} f^{b}(1-f)^{a-1} d f
\end{gathered}
$$

If $a>0$ is real non-integer

$$
(1-f)^{a-1}=\sum_{j=0}^{\infty} \frac{(-1)^{j} \sqrt{(a)} f^{j}}{\sqrt{(a-j)} j !}
$$




$$
\frac{1}{\lambda^{r+1}} \frac{\sum_{j=0}^{\infty}(-1)^{j} \sqrt{(a)}}{\sqrt{(a-j)} j !} \int_{0}^{1} f^{b+j}|\operatorname{Inf}|^{r} d f
$$

Using the fact that

$$
\begin{gathered}
\int_{0}^{1} x^{p}|\operatorname{In} x|^{q} d x=\frac{\overline{(1+q)}}{(1+p)^{q+1}} \text {, we have } \\
\frac{1}{\lambda^{r+1}} \frac{\sum(-1)^{j} \sqrt{(a)}}{\sqrt{(a-j)} j !} \frac{\sqrt{r+1}}{(1+b+j)^{r+1}}
\end{gathered}
$$

Therefore, mgf of $\mathrm{X}$

$$
\begin{aligned}
& M_{(t)}=\frac{\lambda}{\beta(a, b+1)} \sum_{r=0}^{\infty} \frac{t^{r}}{r !} \frac{1}{\lambda^{r+!}} \frac{\sum_{1=\infty}^{\infty}(-1)^{j} \sqrt{(a)}}{\sqrt{(a-j)} j !} \frac{\sqrt{(r+1)}}{(b+j+1)^{r+1}} \\
& =\frac{1}{\lambda^{r}(a, b+1)} \sum_{r=0}^{\infty} \sum_{j=0}^{\infty} \frac{t^{r}(-1)^{j} \sqrt{(a)} \sqrt{(r+1)}}{r ! \sqrt{(a-j)} j !(b+j+1)^{r+1}} \\
& M_{(t)}=\frac{\sqrt{a+b+1}}{\lambda^{r} \sqrt{a} \sqrt{b+1}} \sum_{r=0}^{\infty} \sum_{j=0}^{\infty} \frac{t^{r}(-1)^{j} \sqrt{(a)} \sqrt{(r+1)}}{\overline{(a-j)} j !(b+j+1)^{r+1}} \\
& =\frac{\sqrt{a+b+1}}{\lambda^{r} \sqrt{a} \sqrt{b+1}} \sum_{r=0}^{\infty} \sum_{j=0}^{\infty} \frac{t^{r}(-1)^{j} \sqrt{(a)} \sqrt{(r+1)}}{\sqrt{(a-j)} j !(b+j+1)^{r+1}} \\
& M_{(t)}=\frac{\sqrt{a+b+1}}{\lambda^{r} \sqrt{b+1}} \sum_{r=0}^{\infty} \sum_{j=0}^{\infty} \frac{t^{r}(-1)^{j} \sqrt{(r+1)}}{r ! \sqrt[(a-j)]{(b+j+1)^{r+1} j !}}
\end{aligned}
$$

and for integer $a>0$, we have

$$
M_{(t)}=\frac{1}{\lambda^{r} \beta(a, b+1)} \sum_{r=0}^{\infty} \frac{t^{r}}{r !} \sqrt{(r+1)} \sum_{j=0}^{a-1}\left(\begin{array}{c}
a-1 \\
j
\end{array}\right) \frac{(-1)^{j}}{(b+j+1)^{r+1}} .
$$

Therefore, using the fact that

$$
M_{(t)}=\sum_{r=0}^{\infty} \frac{t^{r}}{r !} \mu_{r}^{\prime} \text {, then }
$$

For non-integer $a$, the $r$ th moment of $\mathrm{X}$ is

$$
E\left(X^{r}\right)=\frac{\sqrt{(a+b+1)}}{\lambda^{r} \sqrt{(b+1)}} \sum_{j=0}^{\infty} \frac{(-1)^{j} \sqrt{(r+1)}}{\sqrt{(a-j)}(b+j+1)^{r+1}}
$$




$$
E\left(X^{r}\right)=\frac{\sqrt{(a+b+1)} \sqrt{(r+1)}}{\left.\lambda^{r}\right)} \sum_{j=0}^{\infty} \frac{(-1)^{j}}{\sqrt{(a-j)}(b+j+1)^{r+1}}
$$

When $a>0$ is integer, we have

$$
E\left(X^{r}\right)=\frac{\sqrt{(r+1)}}{\lambda^{r} \beta(a, b+1)} \sum_{j=0}^{a-1} \frac{\left(\begin{array}{c}
a-1 \\
j
\end{array}\right)(-1)^{j}}{(b+j+1)^{r+1}}
$$

It follows from Eqs. (10) and (11) that when $a=1$ and $b=1$, and $r=1$, it reduces to the mean of power exponential distribution which is

$$
E\left(X^{r}\right)=\frac{2 \longdiv { r + 1 }}{\lambda^{r}}
$$

\section{Estimation of Parameters}

Here we derive the maximum likelihood estimates (MLEs) of the parameters of the Beta Power Exponential Distribution.Let $x_{1}, x_{2}, \ldots, x_{n}$ be a random sample of size $n$ on $X^{\sim} \operatorname{BPED}(a, b, \lambda)$.

The likelihood function for $\theta=(a, b, \lambda)$ may be written as

$$
L=\frac{\lambda^{n}}{[\beta(a, b+1)]^{n}} \prod_{i=1}^{n}\left(1-e^{-\lambda x}\right)^{a-1}\left(e^{-\lambda \sum_{i=1}^{n} x}\right)^{b+1}
$$

The values of the parameters that maximize the likelihood function also maximize the log likelihood. Taking the logarithm of equation ( ), we have

$$
\begin{aligned}
& l=\log L(x \mid a, b, \lambda) \\
& =n \log \lambda-n \log \beta(a, b+1)+(a-1) \sum_{i=1}^{n} \log \left(1-e^{-d x}\right)
\end{aligned}-(b+1) \lambda \sum_{i=1}^{n} x
$$

Since $\beta(a, b+a)=\frac{\sqrt{(a)} \sqrt{(b+1)}}{\sqrt{(a+b+1)}}$

$$
\begin{aligned}
& = n \operatorname { l o g } \lambda - n \operatorname { l o g } \sqrt { ( a ) } - n \operatorname { l o g } \overline { ( b + 1 ) } + n \operatorname { l o g } \longdiv { ( a + b + 1 } \\
& +(a-1) \sum_{i=1}^{n} \log \left(1-e^{-\lambda x}\right)-(b+1) \lambda \sum_{i=1}^{n} x
\end{aligned}
$$

Differentiating Eq. (14) with respect $a, b$ and $\lambda \quad$ respectively, and setting results to zero we have

$$
\frac{\partial l}{\partial a}=\frac{-n \overline{(a)}^{1}}{\sqrt{(a)}}+\frac{n \overline{(a+b+1)}^{1}}{\sqrt{(a+b+1)}}+\sum_{i=1}^{n} \log \left(1-e^{-\lambda x}\right)
$$




$$
\begin{aligned}
& \frac{\partial l}{\partial b}=\frac{-n \overline{(b+1)}^{1}}{\sqrt{(b+1)}}+\frac{n \overline{(a+b+1)}^{1}}{\sqrt{(a+b+1)}}-\lambda \sum_{i=1}^{n} x \\
& \frac{\partial l}{\partial \lambda}=\frac{n}{\lambda}-(b+1) \sum_{i=1}^{n} x+\lambda(a-1) \sum_{i=1}^{n} \frac{e^{-\lambda x}}{1-e^{-\lambda x}}
\end{aligned}
$$

Solving the above equations simultaneously for $a, b$, and $\lambda$ gives us their respective estimates $\hat{a}, \hat{b}$ and $\hat{\lambda}$. Also, finding the derivatives of these equations, gives us the diagonal elements of the Fisher's Information Matrix. They are as follows:

$$
\begin{aligned}
& \frac{\partial^{2} l}{\partial a^{2}}=-n \Psi^{1}(a)+n \Psi^{1}(a+b+1) \\
& \frac{\partial^{2} l}{\partial a^{2}}=-n \Psi^{1}(a+b+1) \\
& \frac{\partial^{2} l}{\partial b^{2}}=-n \Psi^{1}(b+1)+n \Psi^{1}(a+b+1) \\
& \frac{\partial^{2} l}{\partial a \partial \lambda}=\lambda \sum_{i=1}^{n} \frac{e^{-\lambda x}}{1-e^{-\lambda x}} \\
& \frac{\partial^{2} l}{\partial b \partial \lambda}=-\sum_{i=1}^{n} x \\
& \frac{\partial^{2} l}{\partial a^{2}}=(a-1) \sum_{i=1}^{n} \frac{e^{-\lambda x}\left(1-e^{-\lambda x}-\lambda x\right)}{\left(1-e^{-\lambda x}\right)^{2}}
\end{aligned}
$$

Note that $\Psi(k)=\Gamma(k)^{\prime} / \Gamma(k)$ the digamma function.

The Fisher's information Matrix is very useful tool for calculating the interval estimates, asymptotic variances, covariance and tests of hypothesis of $a, b$ and $\lambda$.

\section{Application}

In this section we compare the fit of Beta power exponential distribution to one real data set. The data set is obtained from Smith and Naylor (1987).

The data are the strengths of $1.5 \mathrm{~cm}$ glass fibres, measured at the National Physical Laboratory

England.

The data set is: $0.55,0.93,1.25,1.36,1.49,1.52,1.58,1.61,1.64,1.68,1.73,1.81,2,0.74,1.04,1.27,1.39$, 1.49, 1.53, 1.59, 1.61, 1.66, 1.68, 1.76, 1.82, 2.01,0.77, 1.11, 1.28, 1.42, 1.5, 1.54, 1.6, 1.62, 1.66, 1.69, 1.76, $1.84,2.24,0.81,1.13,1.29,1.48,1.5,1.55,1.61,1.62,1.66,1.7,1.77,1.84,0.84,1.24,1.3,1.48,1.51,1.55$, $1.61,1.63,1.67,1.7,1.78,1.89$.

The descriptive statistics, the MLE of the parameters, the log-likelihood values as well as the Akaike 
information criterion are given in the tables below.

\section{The Qprom of Glass fibre}

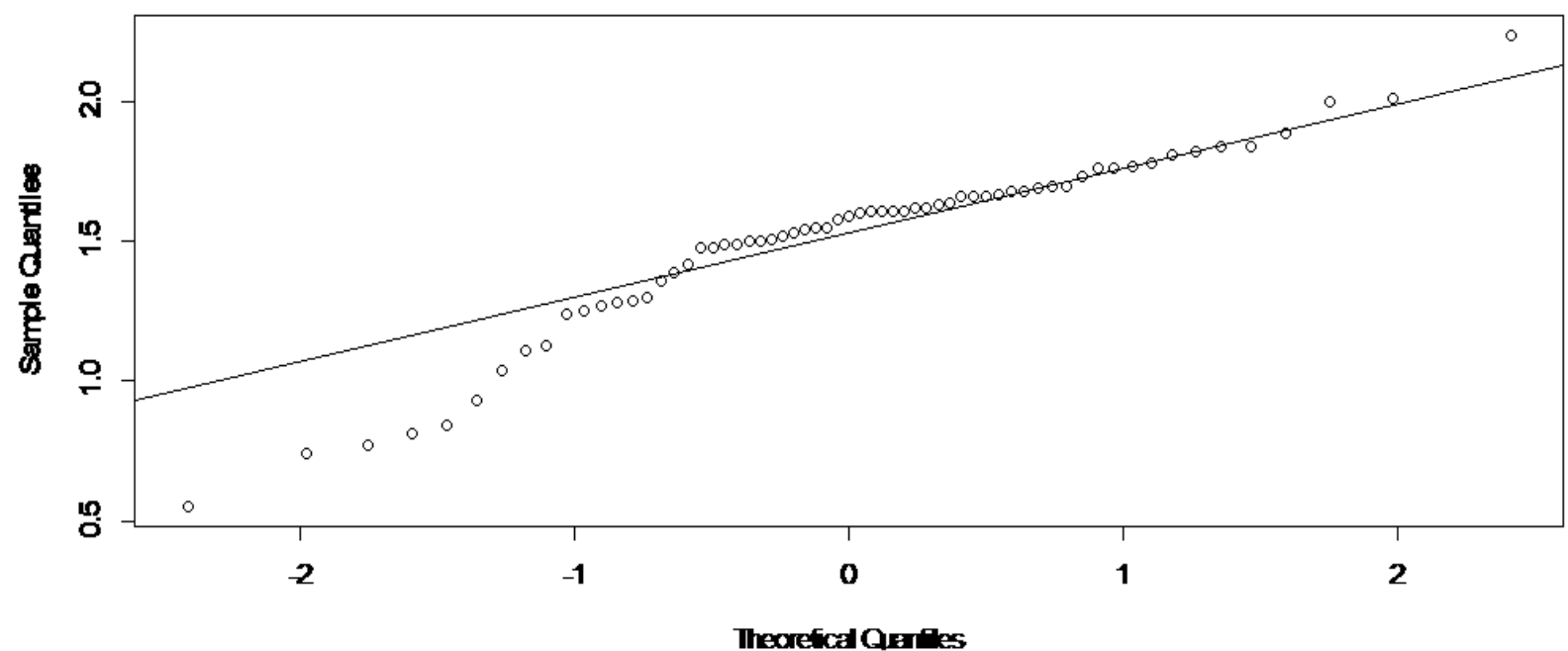

Box plot of Glass fibre

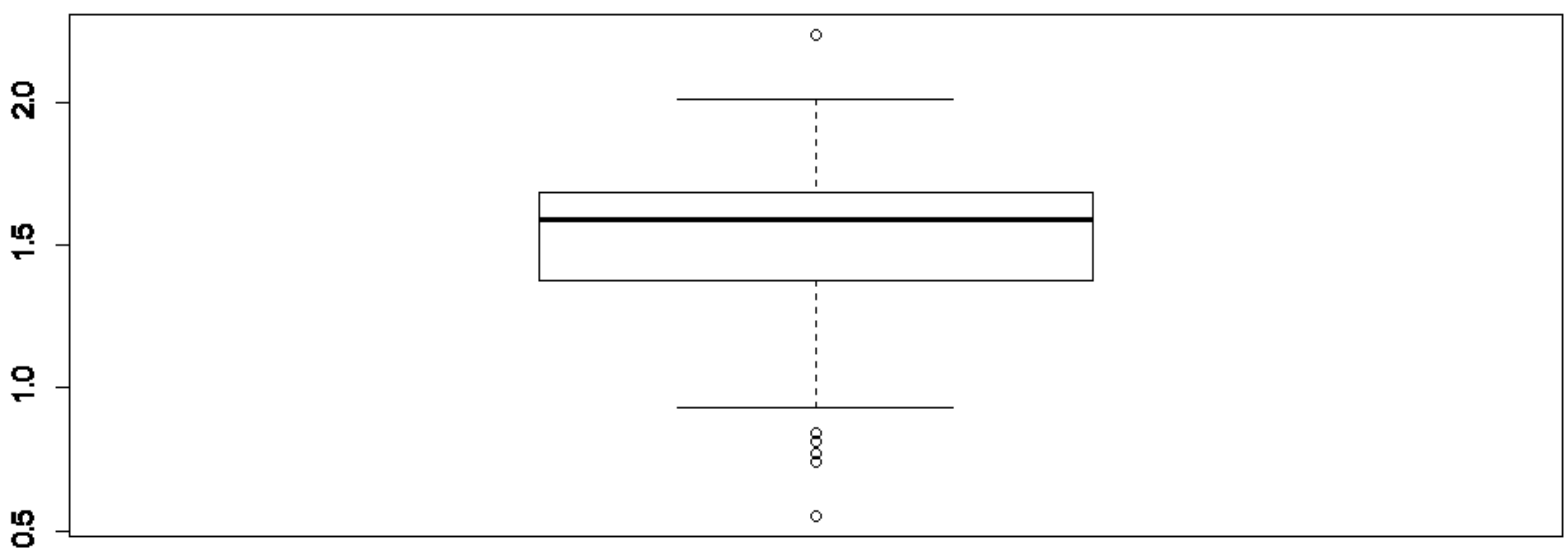

Table 1

Descriptive Statistics

\begin{tabular}{llccccc}
\hline Min & $\mathrm{Q}_{1}$ & $\mathrm{Q}_{2}$ & Mean & $\mathrm{Q}_{3}$ & Max & Var \\
\hline 0.550 & 1.375 & 1.590 & 1.507 & 1.685 & 2.240 & 0.105 \\
\hline
\end{tabular}

Table 2

MLES of the Model parameters, the negative log-likelihood values and the statistics AIC

\begin{tabular}{lllllll}
\hline & Estimates & \multicolumn{5}{c}{ Statistics } \\
\hline Model & $\mathrm{a}$ & $\mathrm{b}$ & $\mathrm{c}$ & $\lambda$ & Likelihood & AIC \\
\hline Beta power exponential & 1.00006 & 1.9999 & 1 & $3.0429 \mathrm{e}-07$ & -572.5149 & 1147.03 \\
Exponentiated exponential & 2.0006 & 1.0006 & 1 & $2.2944 \mathrm{e}-07$ & -611.5369 & 1227.074 \\
\hline
\end{tabular}

Tables 1 and 2 provide some descriptive statistics and the MLES of the model parameters. Since the values of the Akaike information is smaller for the Beta power distribution compared with the exponentiated 
exponential distribution, it

\section{Conclusion}

The existing two parameter power exponential distribution is extended with the introduction of two extra shape parameters giving rise to the Beta-power exponential distribution which has a better shape, broader tails and a class of hazard rate functions depending on the parameters.

Detailed studies of the statistical properties of the proposed distribution which include moments, moment generating function among others have been presented.

The parameters of the model were estimated by method of maximum likelihood which actually paves way for the derivation of fisher information matrix. Real life application indicates that Beta-power exponential distribution apart from its flexibility has better representation of data than exponentiated exponential distribution.

\section{References}

Akinsete, A., Famoye, F., \& Lee, C. (2008). The beta-pareto distribution. Statistics, 42 (6), 547-563

Akinsete, A., Famoye, F., \& Lee, C. (2008). The beta-pareto distribution. Statistics, 42 (6), 547-563.

Cordeiro, G. M., \& de Castro, M. (2011). A new family of generalized distributions. Journal of Statistical Computation and Simulation, 81 (7), 883-898.

Eugene, N. C., \& Famoye, F. (2002). Beta-normal distribution and its applications. Communication in Statistics: Theory and Methods, 31, 497-512.

Famoye, F., Lee, C., \& Olugbenga, O. (2005). The beta-Weibull distribution. Journal of Statistical Theory and Applications, 4 (2), 121-138.

Gupta, R. D. and Kundu, D. (1999). Generalized exponential distributions. Australian and New Zealand Journal of Statistics 41, 173-188.

Gupta, R. D. and Kundu, D. (2001). Exponentiated exponential family: an alternative to gamma and Weibull. Biometrical Journal 43, 117-130

Jones, M. C. (2004). Families of distributions arising from distributions of order statistics. Sociedad de Estadistica e Investigacion Operativa Test, 13 (1), 1- 43.

Kozubowski, T., \& Nadarajah, S. (2008). The beta-Laplace distribution. Journal of Computational Analysis and Applications, 10 (3), 305-318

Nadarajah, S., \& Kotz, S. (2004). The beta-Gumbel distribution. Mathematical Problems in Engineering, 2004 (1), 323-332.

Nadarajah, S., \& Kotz, S. (2006). The beta-exponential distribution. Reliability Engineering and System Safety, 91 (1), 689-697.

Shittu, O.I, Kazeem A. Adepoju (2013).”On the Beta-Nakagami Distribution” (Canada journal of progress in applied Mathematics, vol 5, No1, 2013 